\title{
Variable Rate Space-Time- Block-Code Error Analysis for Ricean Faded Channel for PSK and QAM Signals
}

\author{
Kapil Gupta \\ Department of Electronics \& \\ Communication Engineering, \\ Faculty of Engineering and \\ Technology, Mody Institute of \\ Technology and Science \\ (Deemed University) \\ Lakshmangarh, Dist. Sikar \\ Rajasthan (INDIA)
}

\author{
P. K. Ghosh \\ Department of Electronics \& \\ Communication Engineering, \\ Faculty of Engineering and \\ Technology, Mody Institute of \\ Technology and Science \\ (Deemed University) \\ Lakshmangarh, Dist. Sikar \\ Rajasthan (INDIA)
}

\begin{abstract}
The design of wireless systems is open for further improvements in respect of spectrum efficiency, coverage area and link reliability. Multiple-Input-Multiple-Output (MIMO) links are capable to overcome the limitations of data rate and range of wireless devices by exploiting bandwidth efficiency of multiple transmit and receive antennas. Improved efficiency can further be possible by incorporating the Space Time Block Code (STBC) with MIMO system. Researches on MIMO systems with STBC have becoming an important area that improves the performance of system without additional bandwidth or transmit power requirements. This paper presents STBC models in Ricean fading environment using various combinations of transmit and receive antenna numbers. The simulations results have been obtained in MATLAB platform. The bit error rate performance has been analyzed for the Ricean factors of $\mathrm{k}=0,1$, and 10 in both the BPSK and 16 QAM modulation schemes.
\end{abstract}

\section{Keywords}

Multiple-Input-Multiple-Output (MIMO), Space Time Block Code (STBC), Ricean channel, Maximum Likelihood (ML).

\section{INTRODUCTION}

The MIMO scheme can fulfill the increasing demand of very high speed data communication systems [1]-[2]. In MIMO system, the link between transmitter and receiver is equipped with multiple antennas at both the transmitting as well as at receiving ends [3]. The received signals on the link are combined in such a fashion that the data rate and quality of the system are enhanced. Further, MIMO system can be thought to provide multiple independent channels, so the channel capacity increases linearly with the number of antennas [4]. MIMO systems have proved high spectrum efficiency and channel capacity [5][6]. A well known MIMO system such as V-BLAST architecture operates at very high spectral efficiency with low encoding and decoding complexities [2]. Girish.et.al [7] has shown space-time codes in an optimal Signal-to-Noise Ratio (SNR) framework and proved that they achieve the maximum SNR using Maximal-
Ratio-Combining (MRC) technique. In [8] the authors proposed a new full-rate STBC for two transmit antennas which can be designed to achieve maximum diversity and optimized coding gain and reduced-complexity with Maximum Likelihood (ML) decoding. In multipath propagation, a transmitted signal gets received at the receiver over multiple paths. Due to multipath effect, there is fluctuation in the received signal amplitude, phase, and time of arrival, and this is what is known as multipath fading [9].

Spatial diversity can improve the system performance by combating the effects of multipath fading and thereby increases the reliability of the wireless channel [9]. Transmit diversity can be achieved through Space Time Coding (STC) in multiple antenna systems. It has been proved that Orthogonal Space Time Block Code (OSTBC) [10] provide full diversity with maximum likelihood (ML) decoding technique but limits its maximum achievable code rate less than one, in case the number of transmit antennas are more than two [11]. Additionally, at the transmitter if the channel state information (CSI) is unknown then space-time codes are used and can achieve some diversity gain.

Alamouti introduced a simple but effective diversity scheme in [12] for two transmit antennas and achieved maximum diversity gain but no coding gain with minimum decoding complexity. Using two transmit antenna system and one receive antenna, the scheme provides the same diversity order as Maximal Ratio Receiver Combining (MRRC) [13] with one transmitter antenna and two receive antennas. Tarokh.et.al in [14] describe a new two-dimensional way of encoding and decoding signals transmitted over wireless fading channels using multiple transmit antennas. In [15], the generalized Alamouti scheme was introduced to an arbitrary number of antennas in MIMO with OSTBC technique.

The study of transmit diversity using STBC in MIMO system with ML decoding is an important area of research. This not only improves the diversity order but also reduces decoding complexity. The authors in this article present MIMO system model with STBC using ML detector and show improved diversity gain. 


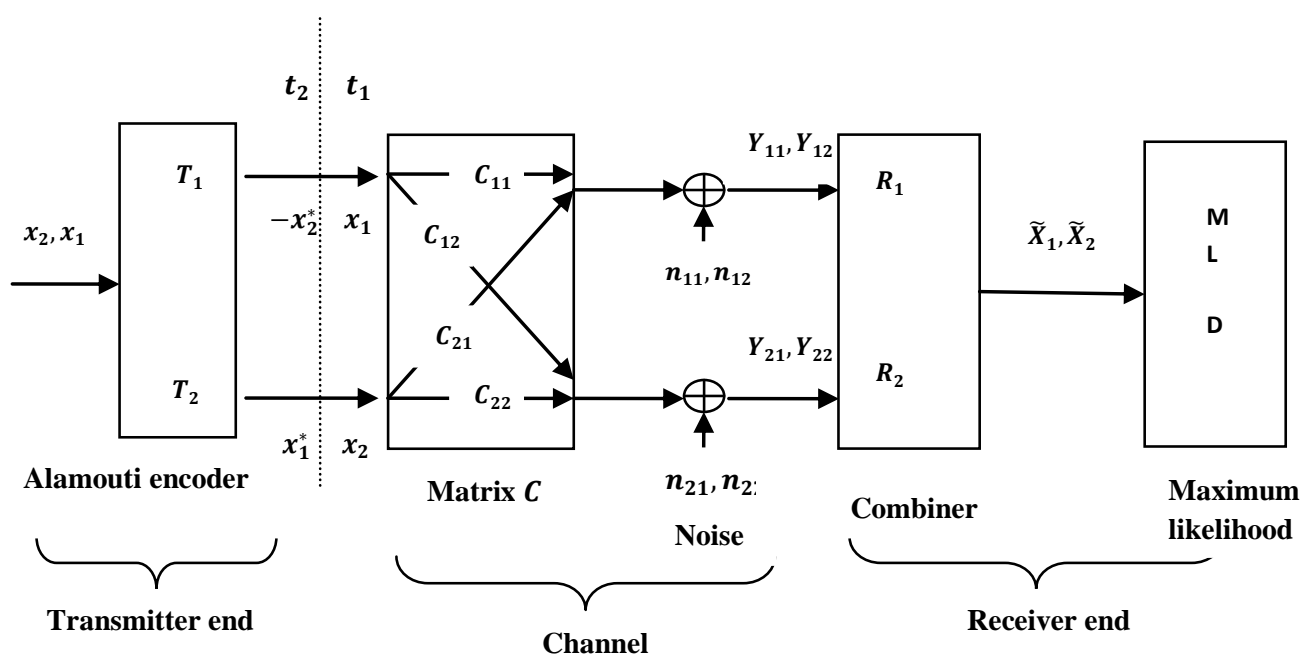

Fig. 1 Block diagram of a 2Tx, 2Rx MIMO system with Alamouti scheme

This paper is organized as follows. Section 2 deals with generalized system model for MIMO system with STBC techniques. Section 3 provides simulation results and performance analysis. Finally, in section 4, conclusions are made.

\section{SYSTEM MODEL}

The elements in MIMO systems are transmitter $\left(T_{x}\right)$, the receiver $\left(R_{x}\right)$ and the channel. The symbols $T_{n}$ and $R_{m}$ denote the number of antenna elements at transmitter and receiver sides, respectively. The system contains space-time encoder at transmission end, space-time decoder with ML detector at the reception end. Thus the MIMO channel consists of $n$ inputs and $m$ output elements, the channel matrix with $R_{m}$ outputs and $T_{n}$ inputs is represented as $\left[\left(R_{m}\right) \times\left(T_{n}\right)\right]$. Each element of the matrix say $C_{i j}$ denotes the path gain or channel transfer function between the $j^{\text {th }}$ transmit antenna and $i^{\text {th }}$ receiver antenna. Assume a quasi-static channel, that is the channel property varies randomly between two bursts but remains fixed within a transmission interval. The channel matrix $\boldsymbol{C}$ with $R_{m}$ outputs and $T_{n}$ inputs can now be represented as

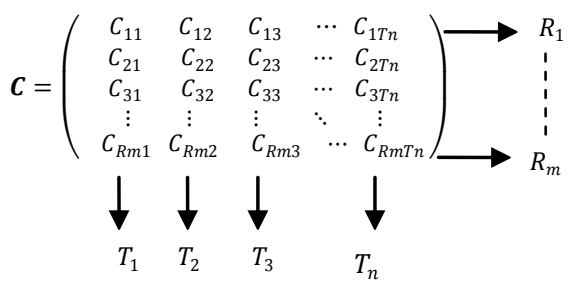

where $C_{i j}$ is the channel transfer function from $j^{\text {th }}$ transmit antenna and the $i^{\text {th }}$ receiver antenna.

A block diagram of a MIMO system for 2 transmitter and 2 receiver antennas with Alamouti scheme is shown in Fig 1. Here a space-time encoder is used at the transmitter which generates symbols to be transmitted at time instant $t_{1}$ and $t_{2}$, where $t_{2}>t_{1}$.

To ensure the prevention of correlation due to spacing, the antennas are typically spaced at least $\frac{\lambda_{c}}{2}$ apart where $\lambda_{c}$ is the carrier wavelength [16]. The encoder transmits symbol $\boldsymbol{X}$ which can be written in matrix form as [12]

$$
\boldsymbol{X}=\left(\begin{array}{rr}
x_{1} & x_{2} \\
-x_{2}^{*} & x_{1}^{*}
\end{array}\right)
$$

There are two time slots for transmitting the symbols. In first time slot $t_{1}$, antenna 1 transmits $x_{1}$ and antenna 2 transmits $x_{2}$ symbol. In the next time slot say $t_{2}$, antenna 1 transmit $-x_{2}^{*}$ and antenna 2 transmits $x_{1}^{*}$ symbol where $x^{*}$ denotes the complex conjugate of $x$.

The MIMO signal model is described as

$Y=C X+N$

where $\boldsymbol{Y}$ is the received vector of order $R_{m} \times 1, \boldsymbol{X}$ is the transmitted vector of order $T_{n} \times 1$ and $\boldsymbol{C}$ is the channel matrix of dimension $\left[R_{m} \times T_{n}\right]$ and $\boldsymbol{N}$ is the noise vector of order $R_{m} \times 1$. Each noise element is modeled as independent identically distributed (i.i.d) white Gaussian noise [16] and variance of $\left(\frac{T_{n}}{2 S N R}\right)$ (that is 0.5 per dimension), where SNR is the signal-to-noise ratio of the channel.

There are two signal channels; one is the line-of-sight (LOS) and the other is non-line-of-sight (NLOS). In this paper we deal with LOS environment only. The channel matrix $\boldsymbol{C}$ for the LOS case in MIMO is given by [17]

$\boldsymbol{C}=\sqrt{\frac{k}{1+k}} \boldsymbol{C}_{L O S}+\sqrt{\frac{1}{1+k}} \boldsymbol{C}_{N L O S}$

where $k=\frac{P_{L O S}}{P_{N L O S}}, P_{L O S}$ is power in LOS component and $P_{N L O S}$ is the power in NLOS component and $\boldsymbol{C}_{L O S}, \boldsymbol{C}_{N L O S}$ refer to rank-one matrices corresponding to the LOS and NLOS component, respectively. The power of LOS and NLOS component should satisfy the $P_{L O S}+P_{N L O S}=1$.

The $\boldsymbol{C}_{N L O S}$ component is modeled as [4]

$C_{i j}=\sqrt{\frac{1}{2}}(\operatorname{Normal}(0,1)+\sqrt{-1} \operatorname{Normal}(0,1))$

However, for the case of multipath, LOS component rarely dominates but we have simulated the results for both LOS and NLOS dominating components. The output of the combiner at the receiver, which is also the input to the ML detector, has the form 


$$
\widetilde{X}=C^{H} \boldsymbol{Y}
$$

where $\boldsymbol{C}^{\boldsymbol{H}}$ is the transpose conjugate of $\boldsymbol{C}$. By substituting eq. (3) in eq. (6) yields $\widetilde{\boldsymbol{X}}=\boldsymbol{C}^{\boldsymbol{H}}(\boldsymbol{C} \boldsymbol{X}+\boldsymbol{N})$ which can be written as

$$
\widetilde{X}=\|C\|_{F}^{2} I_{2} X+C^{H} N
$$

where \|\|$_{F}$ is the Frobenius norm. The outputs of the combiner are $\tilde{X}_{1}=\|C\|_{F}^{2} X_{1}+\widetilde{N}_{1}$ and $\widetilde{X}_{2}=\|C\|_{F}^{2} X_{2}+\widetilde{N}_{2}$, indicating that the symbol $\tilde{X}_{1}$ is a function of $X_{1}$ and $\tilde{X}_{2}$ is a function of $X_{2}$ only, and $\widetilde{N}_{1}$ and $\widetilde{N}_{2}$ are the decoupled noise components. Thus, the STBC and decoding combined together transforms the fading MIMO channel into Gaussian SISO channel with effective channel gain of $\|C\|_{F}$.

\subsection{Alamouti STBC}

Alamouti proposed a technique on transmit diversity in [12]. The STBC scheme proposed by Alamouti uses two antenna at the transmitter end and $R_{m}$ antennas at the receiver end. This scheme is of full rate since it transmits 2 symbols at every 2 time intervals and capable of achieving maximum diversity order of $2 R_{m}$. The Alamouti encoding scheme can be modeled by the matrix given as



The row of each coding scheme represents a time slot, while the column represents the transmitted symbol from different antennas. The first column elements of both the columns, namely symbols $x_{1}$ and $x_{2}$ are transmitted simultaneously during first time slot $t_{1}$ by antenna 1 and 2 respectively. At next time slot $t_{2}$, symbol $-x_{2}^{*}$ is transmitted by antenna 1 and symbol $x_{1}^{*}$ is transmitted by antenna 2 .

For one receiver antenna case the received equation can be written as

$Y_{11}=y_{1}\left(t_{1}\right)=C_{11} x_{1}+C_{12} x_{2}+n_{11}$

$Y_{12}=y_{1}\left(t_{2}\right)=-C_{11} x_{2}^{*}+C_{12} x_{1}^{*}+n_{12}$

$y_{1}\left(t_{1}\right)$ at time instant $t_{1}$ and $y_{1}\left(t_{2}\right)$ at time instant $t_{2}$, at the receiver antenna. Where $Y_{11}$ is the received signal at the receiver end, $n_{i j}$ is the additive noise for the $i^{\text {th }}$ receiver antenna at the $j^{\text {th }}$ time slot.

The inputs to the decoder are

$\tilde{X}_{1}=C_{11}^{*} Y_{11}+C_{12} Y_{12}^{*}$

$\tilde{X}_{2}=C_{12}^{*} Y_{11}+C_{11} Y_{12}^{*}$.

By substituting Eq. (9) and conjugate of Eq. (10) in Eq. (11) gives

$\tilde{X}_{1}=C_{11}^{*}\left(C_{11} x_{1}+C_{12} x_{2}+n_{11}\right)+C_{12}\left(-C_{11} x_{2}^{*}+C_{12} x_{1}^{*}+\right.$

$\left.n_{12}\right)^{*}$

$=C_{11}^{*} C_{11} x_{1}+C_{11}^{*} C_{12} x_{2}+C_{11}^{*} n_{11}+C_{12}^{*} C_{12} x_{1}^{*}-C_{11}^{*} C_{12} x_{2}+$

$C_{12} n_{12}^{*}$

$=\left(\alpha_{11}^{2}+\alpha_{12}^{2}\right) x_{1}+C_{11}^{*} n_{11}+C_{12} n_{12}^{*}$

Similarly for $\tilde{X}_{2}$ we get:

$\tilde{X}_{2}=\left(\alpha_{11}^{2}+\alpha_{12}^{2}\right) x_{2}-C_{11} n_{12}^{*}+C_{12}^{*} n_{11}$
In the above equations (13) and (14), $\alpha_{i j}^{2}$ is the magnitude of the channel transfer function $C_{i j}$. The output symbols $\tilde{X}_{1}$ and $\tilde{X}_{2}$ (vide Fig. 2) of the combiner are then sent to a maximum likelihood (ML) detector to estimate the transmitted symbol $x_{1}$ and $x_{2}$. The ML detector decodes in favor of symbol $x_{1}$ if the value of the following condition

$$
\begin{aligned}
& \left|\left(\sum_{i=1}^{M_{r}} Y_{i 1} C_{i 1}^{*}+Y_{i 2}^{*} C_{i 2}\right)-x_{1}\right|^{2}+ \\
& \left(-1+\sum_{i=1}^{M_{r}} \sum_{j=1}^{N_{t}}\left|C_{i j}\right|^{2}\right)\left|x_{1}\right|^{2} \\
& \text { is minimum than } \\
& \left|\left(\sum_{i=1}^{M_{r}} Y_{i 1} C_{i 2}^{*}-Y_{i 2}^{*} C_{i 1}\right)-x_{2}\right|^{2}+\left(-1+\sum_{i=1}^{M_{r}} \sum_{j=1}^{N_{t}}\left|C_{i j}\right|^{2}\right)\left|x_{2}\right|^{2}
\end{aligned}
$$

Otherwise decision will go in favor of $x_{2}$.

For two receiver antenna case the received equations are as follows:

$Y_{11}=y_{1}\left(t_{1}\right)=C_{11} x_{1}+C_{12} x_{2}+n_{11}$

at time instant $t_{1}$,by first antenna at receiver,

$Y_{12}=y_{1}\left(t_{2}\right)=-C_{11} x_{2}^{*}+C_{12} x_{1}^{*}+n_{12}$

at time instant $t_{2}$,by first antenna at receiver,

$Y_{21}=y_{2}\left(t_{1}\right)=C_{21} x_{1}+C_{22} x_{2}+n_{21}$

at time instant $t_{1}$,by second antenna at receiver,

$Y_{22}=y_{2}\left(t_{2}\right)=-C_{21} x_{2}^{*}+C_{22} x_{1}^{*}+n_{22}$

at time instant $t_{2}$, by second antenna at receiver.

In this case the input to the decoder is

$\tilde{X}_{1}=C_{11}^{*} Y_{11}+C_{12} Y_{12}^{*}+C_{21}^{*} Y_{21}+C_{22} Y_{22}^{*}$

and $\tilde{X}_{2}=C_{12}^{*} Y_{11}+C_{11} Y_{12}^{*}+C_{22}^{*} Y_{21}+C_{11} Y_{22}^{*}$

Now substituting Eq. (17), Eq. (19) and conjugate of Eq. (18), Eq. (20) in Eq. (21) and in Eq. (22) yields

$\tilde{X}_{1}=\left(\alpha_{11}^{2}+\alpha_{12}^{2}+\alpha_{21}^{2}+\alpha_{22}^{2}\right) x_{1}+C_{11}^{*} n_{11}+C_{12} n_{12}^{*}+$
$C_{21}^{*} n_{21}+C_{22} n_{22}^{*}$
and
$\tilde{X}_{2}=\left(\alpha_{11}^{2}+\alpha_{12}^{2}+\alpha_{21}^{2}+\alpha_{22}^{2}\right) x_{2}-C_{11} n_{12}^{*}+C_{12}^{*} n_{11}+$
$C_{22}^{*} n_{21}-C_{21} n_{22}^{*}$

Note that there is no requirement of CSI at the transmitter in Alamouti STBC, and it can acquire full diversity of two even when 2 transmit antennas and 1 receive antenna are used. This scheme can be used with 2 transmit antennas and $R_{m}$ receive antennas while having a full diversity of $2 R_{m}$.

\subsection{Space Time Block Code (Orthogonal)}

In [11] real and complex orthogonal codes are designed to achieve full diversity and full rate code is obtained for the case of real orthogonal codes. Alamouti scheme is a special condition of general orthogonal space time block codes (OSTBC's). There are four symbols $x_{1}, x_{2}, x_{3}$ and $x_{4}$; these symbols are transmitted in 8 time slots and so its rate becomes half. The transmit matrix $\boldsymbol{X}$ for OSTBC for $T_{n}=3$ and rate $1 / 2$ is given by [15].

The row represents time slots; there are 8 time slot in this matrix. In a time slot, one symbol is transmitted by each transmitter antenna. Four symbols and their conjugate are being transmitted in 8 time slots. The ML detector decodes in favor of the symbol out of $x_{1}, x_{2}, x_{3}$ and $x_{4}$, which fulfils minimum value criteria of Eqs. (26) to (29). 
$\boldsymbol{X}=\left(\begin{array}{ccc}x_{1} & x_{2} & x_{3} \\ -x_{2} & x_{1} & -x_{4} \\ -x_{3} & x_{4} & x_{1} \\ -x_{4} & -x_{3} & x_{2} \\ x_{1}^{*} & x_{2}^{*} & x_{3}^{*} \\ -x_{2}^{*} & x_{1}^{*} & -x_{4}^{*} \\ -x_{3}^{*} & x_{4}^{*} & x_{1}^{*} \\ -x_{4}^{*} & -x_{3}^{*} & x_{2}^{*}\end{array}\right)$

The expressions corresponding to $x_{1}, x_{2}, x_{3}$ and $x_{4}$ symbols are given in Eqs. (26) to (29) respectively.

$\mid\left(\sum_{i=1}^{M_{r}} Y_{i 1} C_{i 1}^{*}+Y_{i 2} C_{i 2}^{*}+Y_{i 3} C_{i 3}^{*}+Y_{i 5}^{*} C_{i 1}+Y_{i 6}^{*} C_{i 2}+Y_{i 7}^{*} C_{i 3}\right)-$ $\left.x_{1}\right|^{2}+\left(-1+2 \sum_{i=1}^{M_{r}} \sum_{j=1}^{N_{t}}\left|C_{i j}\right|^{2}\right)\left|x_{1}\right|^{2}$

If this attains a minimum among other 3 conditions, then symbol $x_{1}$ is detected. Similarly when the expression

$\mid\left(\sum_{i=1}^{M_{r}} Y_{i 1} C_{i 2}^{*}-Y_{i 2} C_{i 1}^{*}+Y_{i 4} C_{i 3}^{*}+Y_{i 5}^{*} C_{i 2}-Y_{i 6}^{*} C_{i 1}+Y_{i 8}^{*} C_{i 3}\right)-$

$\left.x_{2}\right|^{2}+\left(-1+2 \sum_{i=1}^{M_{r}} \sum_{j=1}^{N_{t}}\left|C_{i j}\right|^{2}\right)\left|x_{2}\right|^{2}$

is minimum among other 3 equations, then symbol $x_{2}$ is detected. Likewise, the decision will be in favour of $x_{3}$ if $\mid\left(\sum_{i=1}^{M_{r}} Y_{i 1} C_{i 3}^{*}-Y_{i 3} C_{i 1}^{*}-Y_{i 4} C_{i 2}^{*}+Y_{i 5}^{*} C_{i 3}-Y_{i 7}^{*} C_{i 1}-Y_{i 8}^{*} C_{i 2}\right)-$ $\left.x_{3}\right|^{2}+\left(-1+2 \sum_{i=1}^{M_{r}} \sum_{j=1}^{N_{t}}\left|C_{i j}\right|^{2}\right)\left|x_{3}\right|^{2}$

attains a minimum value. Finally, if $\mid\left(\sum_{i=1}^{M_{r}}-Y_{i 2} C_{i 3}^{*}+Y_{i 3} C_{i 2}^{*}-Y_{i 4} C_{i 1}^{*}-Y_{i 6}^{*} C_{i 3}+Y_{i 7}^{*} C_{i 2}-\right.$ $\left.Y_{i 8}^{*} C_{i 1}\right)-\left.x_{4}\right|^{2}+\left(-1+2 \sum_{i=1}^{M_{r}} \sum_{j=1}^{N_{t}}\left|C_{i j}\right|^{2}\right)\left|x_{4}\right|^{2}$

becomes a minimum among above 3 conditions, then symbol $x_{4}$ is detected.

\section{RESULTS}

Simulated results are obtained for the following cases. Two transmitter-one receiver $(2 \mathrm{Tx}-1 \mathrm{Rx})$, two transmitter-two receiver $(2 \mathrm{Tx}-2 \mathrm{Rx})$, three transmitter - one receiver (3Tx$1 \mathrm{Rx}$ ), and three transmitter-two receiver (3Tx-2Rx) in BPSK and 16 QAM modulation techniques with Ricean factor $\mathrm{k}=0,1$, and 10 . The simulation results are compared for the STBC-MIMO system over Ricean fading channel. For both the STBC codes (Alamouti and orthogonal STBC rate 1/2), the equivalent channel matrix $C$ is computed to determine the combined symbols $\tilde{X}_{1}$ and $\tilde{X}_{2}$.

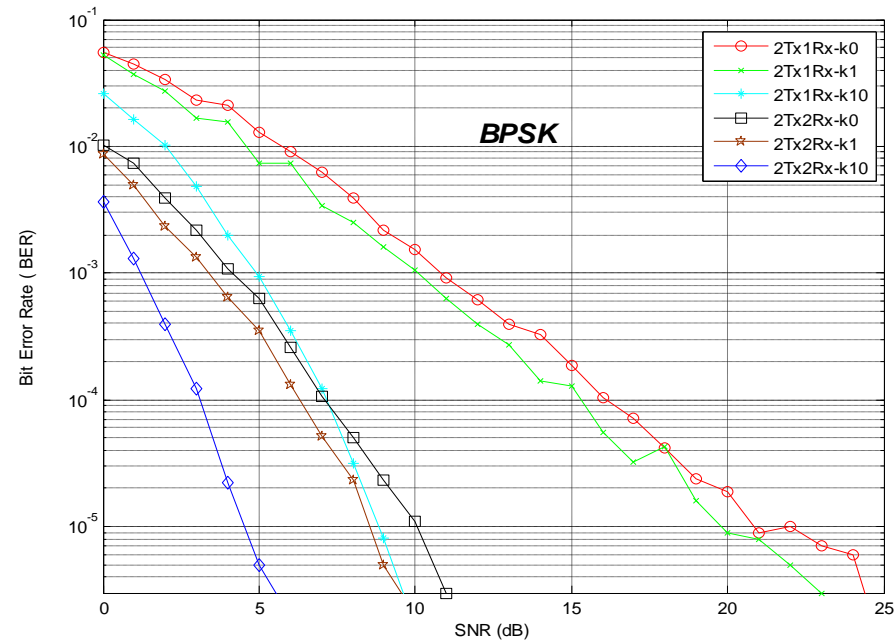

Fig. 2 BER versus SNR for Ricean factor $k=0,1$ \& 10 of OSTBC for $T_{n}=2, R_{m}=1 \& 2$.
The plots of BER versus received SNR for $2 T x-1 R x$ and $2 \mathrm{Tx}-2 \mathrm{Rx}$ are shown in Fig 2 for three Ricean factors of 0,1 and 10. Since Ricean distribution behaves a Rayleigh one for $\mathrm{k}=0$, we get the BER of Rayleigh channel directly for $\mathrm{k}=0$.

For $2 \mathrm{Tx}-1 \mathrm{Rx}$ case, the curves for $\mathrm{k}=10$ and $\mathrm{k}=1$ show diversity gains of $14 \mathrm{~dB}$ and $1.2 \mathrm{~dB}$, respectively measured with respect to the Rayleigh channel $(\mathrm{k}=0)$. The same figures for $2 \mathrm{Tx}-2 \mathrm{Rx}$ case are $5.7 \mathrm{~dB}$ and $1.1 \mathrm{~dB}$, respectively. We observe that $2 \mathrm{Tx}-1 \mathrm{Rx}$ at $\mathrm{k}=10$ offers better performance than that of $2 \mathrm{Tx}-2 \mathrm{Rx}$ at $\mathrm{k}=0$ for higher SNR values. The $2 T x-2 R x$ case outperforms $2 T x-1 R x$ case

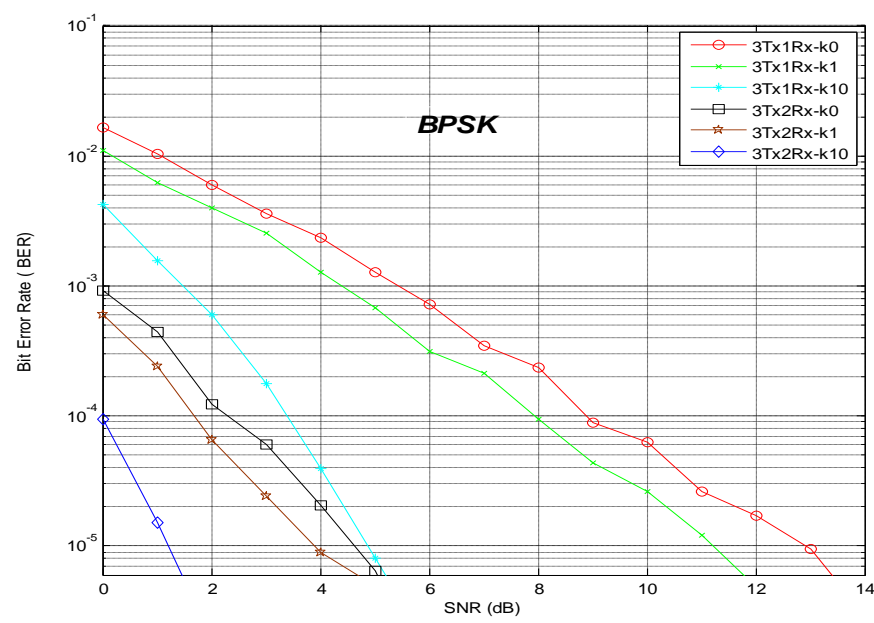

Fig. 3 BER versus SNR for Ricean factor $k=0,1$ \& 10 of OSTBC for $T_{n}=3, R_{m}=1 \& 2$.

Fig 3 shows the plots of BER versus SNR (dB) for 3Tx-1Rx and $3 T x-2 R x$ for Ricean factors of 0,1 and 10 . For 3Tx-1Rx case, the curves for $\mathrm{k}=10$ and $\mathrm{k}=1$ show diversity gains of $8.2 \mathrm{~dB}$ and $1.8 \mathrm{~dB}$, respectively measured with respect to the Rayleigh channel $(\mathrm{k}=0)$. The same for $2 \mathrm{Tx}-2 \mathrm{Rx}$ case are $3.5 \mathrm{~dB}$ and $0.5 \mathrm{~dB}$, respectively. We observe that $3 \mathrm{Tx}-1 \mathrm{Rx}$ has better performance than $2 \mathrm{Tx}-1 \mathrm{Rx}$ for all the values of Ricean factor $\mathrm{k}$. Performance is found to improve by increasing the number of antenna at the transmitter end while keeping receiver antenna fixed.

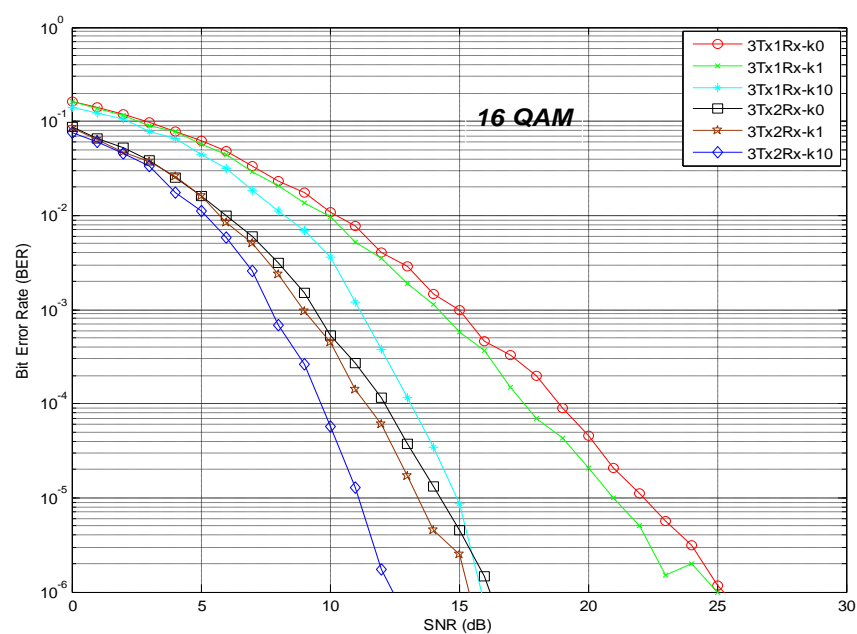

Fig. 4 BER versus SNR for Ricean factor $k=0,1$ \& 10 of OSTBC for $T_{n}=3, R_{m}=1 \& 2$. 
Fig 4 depicts the variation of BER with SNR for 16-QAM modulation scheme in orthogonal STBC applied in Ricean channel. Simulations are performed for three values of Ricean parameters, namely $\mathrm{k}=0,1$, and 10 . Diversity gain of $1.5 \mathrm{~dB}$ and $9 \mathrm{~dB}$ are achieved for $3 \mathrm{Tx}-1 \mathrm{Rx}$ setup and for $3 \mathrm{Tx}-$ $2 \mathrm{Rx}$ diversity gains are $1 \mathrm{~dB}$ and $3.5 \mathrm{~dB}$ for $\mathrm{k}=1 \& 10$ respectively over $\mathrm{k}=0$. In general better performance can be achieved if the number of receiver antennas increase by keeping transmitter antenna fixed and as such, $3 T x-2 R x$ has better performance than $3 \mathrm{Tx}-1 \mathrm{Rx}$.

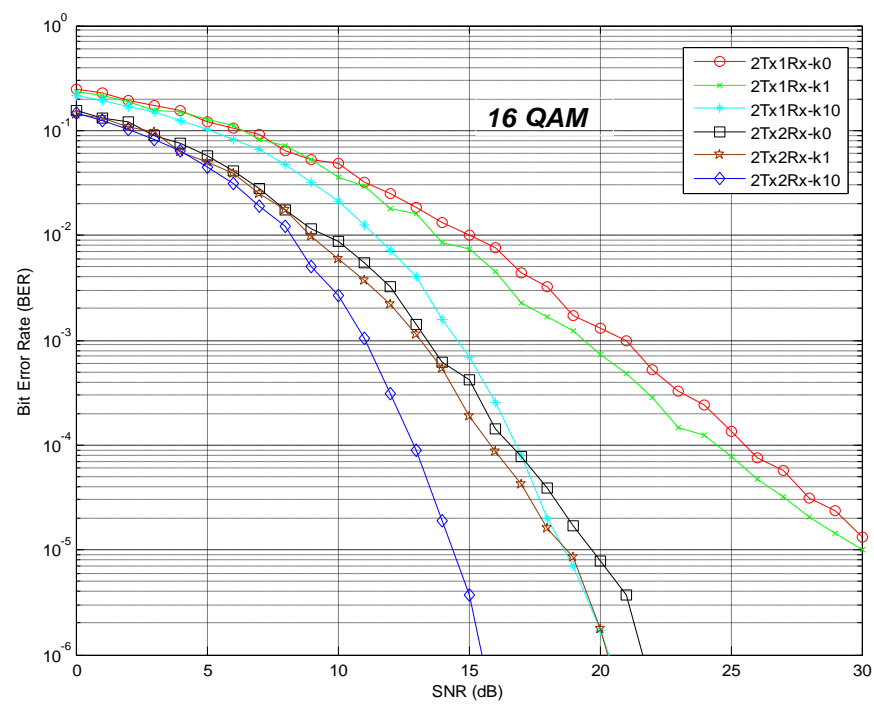

Fig. 5 BER versus SNR for Ricean factor $k=0,1$ \& 10 of OSTBC for $T_{n}=2, R_{m}=1 \& 2$.

The curves are plotted in Fig. 5 for $2 \mathrm{Tx}-1 \mathrm{Rx}$ and $2 \mathrm{Tx}-2 \mathrm{Rx}$ system when $\mathrm{k}=0,1 \& 10$ for 16 -QAM schemes. For $\mathrm{k}=10$, a diversity gain of $11.2 \mathrm{~dB}$ is achieve over $\mathrm{k}=1$ for $2 \mathrm{Tx}-1 \mathrm{Rx}$ and for $2 \mathrm{Tx}-2 \mathrm{Rx}$ case, $\mathrm{k}=10$ and $\mathrm{k}=1$ show a diversity gain of $6.3 \mathrm{~dB}$ and $1.8 \mathrm{~dB}$, respectively. The performance is degraded in comparison with same combination of transmitter and receiver with BPSK modulation.



Fig. 6. BER versus SNR for Ricean factor $k=10$ of OSTBC for $T_{n}=2 \& 3, R_{m}=2$.
A comparison of BPSK and 16 QAM modulation techniques for $2 T x-2 R x$ and $3 T x-2 R x$ is shown in Fig 6 for a fixed value of $\mathrm{k}=10$. As expected BPSK scheme has better performance than 16 QAM in both the combination of transmit and receive antennas. 3Tx-2Rx configuration shows some diversity gain over 2Tx-2Rx. In 16 QAM, 3Tx-2Rx has a diversity gain of 3 $\mathrm{dB}$ over $2 \mathrm{Tx}-2 \mathrm{Rx}$ and for BPSK this gain is about $3.7 \mathrm{~dB}$.

\section{CONCLUSION}

In this paper, we have presented the simulation results of the average BER performance of MIMO space-time block codes, using BPSK and 16 QAM modulation schemes, over Ricean fading channels. We have reviewed the encoding and decoding process for the Alamouti code and OSTBC of rate half. Simulation results are provided to compare the performances of different STBC codes for different combination for numbers of transmit and receive antennas in Ricean fading environment. Also, significant gains can be achieved by increasing the number of transmit antennas. Simulated results indicate that increasing the number of antennas at transmitter side will achieve better BER performances. The increase in Ricean parameter ' $\mathrm{k}$ ' will definitely corresponds to betterment in the performance of the system.

\section{REFERENCES}

[1] David, G., Mansoor, S., Da-shan, S., Smith, P. J., and Ayman, N., 2003. "From Theory to Practice: An Overview of MIMO Space-Time Coded Wireless Systems", IEEE Journal on selected areas in communications, vol. 21, no. 3, pp. 281-302.

[2] Golden, G. D., Foschini, G. J., Valenzuela, R. A., and Wolniasky, P. W., 1999. "Detection algorithm and initial laboratory results using V-BLAST space time communication architecture," IEE Electron. Lett., vol 35, pp. 14-16.

[3] Stoian, R. and Perişoară, L. A. 2006. "Information capacity for a class of MIMO systems," in Proc. 10th WSEAS International Conference on Communications, Athens, Greece, July 13-15, pp. 346-349.

[4] Foschini and M. Gans, "On limits of wireless communications in a fading environment when using multiple antennas," Wireless personal communications, vol. 6, no. 3, pp. 311-335, 1998.

[5] Winters, J. 1987. "On the capacity of radio communication systems with diversity in a Rayleigh fading environment," IEEE Journal on Selected Areas in Communications, vol. 5, no. 5, pp. 871-878.

[6] Maaref, A. and Issa, S. A. 2005. "Capacity of space-time block codes in MIMO Rayleigh fading channels with adaptive transmission and estimation errors," IEEE Trans. Wireless Commun., vol. 4, no. 5, pp. 2568-2578.

[7] Ganesan, G. and Stoica, P. 2001. "Space-time block codes: A maximum SNR approach," IEEE Trans. Inform.Theory, vol. 47, no. 4, pp. 1650-1656.

[8] Rabiei, P., Al-Dhahir, N., and Calderbank, R. 2009. "New Rate-2 STBC Design for 2 TX with ReducedComplexity Maximum Likelihood Decoding" , IEEE Transactions on wireless communications, vol. 8 , no. 2 , pp. 1-11.

[9] Andrea Goldsmith, 2005. "Wireless Communications", Stanford University, Cambridge University Press. 
[10] Wu, J. and Gary, J. S., 2007. "Orthogonal Space-time Block Code Over Time-varying Flat-fading Channels: Channel Estimation, Detection, and Performance Analysis", IEEE Transactions on Communications, vol. 55 , no. 5 .

[11] Tarokh, V., Jafarkhani, H., and Calderbank, A., 1999. "Space-time block codes from orthogonal designs," IEEE Transactions on Information Theory, vol. 45, no. 5, pp. $1456-1467$.

[12] Alamouti, S. 1998. "A simple transmit diversity technique for wireless communications," IEEE Journal on selected areas in communications, vol. 16 , no. 8 , pp. $1451-1458$

[13] Marcelo, M. C. and Garcia, J. J. 2006. "Analytical modeling of ad-hoc networks that utilize space time coding", 4th International Symposium on AdHoc and Wireless Networks, IEEE.

[14] Tarokh, V., Seshadri, N., and Calderbank, A. 1998. "Space-time codes for high data rate wireless communication: performance criterion and code construction," IEEE Transaction on Information Theory, vol. 44, pp. 744-765.

[15] Tarokh, V., Seshadri, N., and Calderbank, A. 1999. "Space-time block coding for wireless communications: performance results," IEEE Journal on selected areas in communications, vol. 17 , no. 3, pp. 451-460.

[16] A. Molisch, 2005. Wireless Communications. WileyIEEE Press.

[17] T. Kaiser, 2005. Smart Antennas-State of the Art. Hindawi Publishing Corporation. 